ARTICLE

DOI: $10.1038 / \mathrm{s} 41467-018-05143-2$

\title{
Fe-N system at high pressure reveals a compound featuring polymeric nitrogen chains
}

\author{
M. Bykov (1) 1, E. Bykova (1) 1,2, G. Aprilis (10 3 , K. Glazyrin², E. Koemets ${ }^{1}$, I. Chuvashova (1) 1,3, I. Kupenko ${ }^{4}$, \\ C. McCammon (10 1, M. Mezouar (1) 5 , V. Prakapenka ${ }^{6}$, H.-P. Liermann², F. Tasnádi 7,8, A.V. Ponomareva ${ }^{8}$, \\ I.A. Abrikosov ${ }^{7}$, N. Dubrovinskaia (i) ${ }^{3} \&$ L. Dubrovinsky (i) ${ }^{1}$
}

Poly-nitrogen compounds have been considered as potential high energy density materials for a long time due to the large number of energetic $\mathrm{N}-\mathrm{N}$ or $\mathrm{N}=\mathrm{N}$ bonds. In most cases high nitrogen content and stability at ambient conditions are mutually exclusive, thereby making the synthesis of such materials challenging. One way to stabilize such compounds is the application of high pressure. Here, through a direct reaction between Fe and $\mathrm{N}_{2}$ in a laserheated diamond anvil cell, we synthesize three ironnitrogen compounds $\mathrm{Fe}_{3} \mathrm{~N}_{2}, \mathrm{FeN}_{2}$ and $\mathrm{FeN}_{4}$. Their crystal structures are revealed by single-crystal synchrotron $\mathrm{X}$-ray diffraction. $\mathrm{Fe}_{3} \mathrm{~N}_{2}$, synthesized at $50 \mathrm{GPa}$, is isostructural to chromium carbide $\mathrm{Cr}_{3} \mathrm{C}_{2}$. FeN $\mathrm{N}_{2}$ has a marcasite structure type and features covalently bonded dinitrogen units in its crystal structure. $\mathrm{FeN}_{4}$, synthesized at $106 \mathrm{GPa}$, features polymeric nitrogen chains of $\left[\mathrm{N}_{4}{ }^{2-}\right]_{n}$ units. Based on results of structural studies and theoretical analysis, $\left[\mathrm{N}_{4}{ }^{2-}\right]_{n}$ units in this compound reveal catena-poly[tetraz-1-ene-1,4-diyl] anions.

\footnotetext{
${ }^{1}$ Bayerisches Geoinstitut, University of Bayreuth, 95440 Bayreuth, Germany. ${ }^{2}$ Photon Science, Deutsches Elektronen-Synchrotron, Notkestrasse 85,22607 Hamburg, Germany. ${ }^{3}$ Material Physics and Technology at Extreme Conditions, Laboratory of Crystallography, University of Bayreuth, 95440 Bayreuth, Germany. ${ }^{4}$ Institut für Mineralogie, University of Münster, Corrensstraße 24, 48149 Münster, Germany. ${ }^{5}$ European Synchrotron Radiation Facility, BP 220, 38043 Grenoble Cedex, France. ${ }^{6}$ Center for Advanced Radiation Sources, University of Chicago, 9700 South Cass Avenue, Argonne, IL 60437, USA. ${ }^{7}$ Department of Physics, Chemistry and Biology (IFM), Linköping University, SE-58183 Linköping, Sweden. ${ }^{8}$ Materials Modeling and Development Laboratory, National University of Science and Technology 'MISIS', Moscow 119049, Russia. Correspondence and requests for materials should be addressed to M.B.(email: maks.byk@gmail.com)
} 
S ince the discovery of a single-bonded cubic nitrogen polymorph $(c g-\mathrm{N})^{1}$, many experimental and theoretical studies were dedicated to the search for high energy density nitrogen allotropes and nitrides ${ }^{2-4}$. Polymeric nitrogen solids have been regarded as the best high energy density materials $(\mathrm{HEDMs})^{5-7}$ owing to the remarkable difference in the average bond energy between the single $\mathrm{N}-\mathrm{N}$ bond $\left(160 \mathrm{~kJ} \mathrm{~mol}^{-1}\right)$, the double $\mathrm{N}=\mathrm{N}$ bond $\left(418 \mathrm{~kJ} \mathrm{~mol}^{-1}\right)$, and the triple $\mathrm{N} \equiv \mathrm{N}$ bond $\left(945 \mathrm{~kJ} \mathrm{~mol}^{-1}\right)^{8}$. A number of single-bonded nitrogen allotropes were predicted to exist at pressures higher than the synthesis pressure of $c g-\mathrm{N}^{9-11}$. However, in the absence of detailed structural information (e.g., single crystal data) about even those single-bonded nitrogen allotropes which were reported to exist ${ }^{1,10}$ any discussion regarding organization of chemical bonding in nitrogen-based HEDMs is difficult. Moreover, it is highly desired to synthesize and stabilize HEDMs at pressures significantly lower than $100 \mathrm{GPa}$, preferably close to ambient.

Numerous studies suggest that polymeric nitrogen networks may be stabilized at lower pressures in compounds ${ }^{4,12,13}$. Theoretical calculations predict existence of different polynitrides $\mathrm{MN}_{\mathrm{x}}$ $(\mathrm{M}=\mathrm{Li}, \mathrm{Be}, \mathrm{Na}, \mathrm{Mg}, \mathrm{Al}, \mathrm{K}, \mathrm{Ca}, \mathrm{Ti}, \mathrm{Cr}, \mathrm{Rb}, \mathrm{Ru}, \mathrm{Cs}, \mathrm{Hf}, \mathrm{W}, \mathrm{Re}, \mathrm{Os}$ $\mathrm{x}=3-10)^{14-31}$ featuring various polymeric nitrogen chains, $\mathrm{N}_{5}$ or $\mathrm{N}_{6}$ rings or even more complex nitrogen networks (e.g., planar $\mathrm{N}_{18}$ rings in $\mathrm{KN}_{8}{ }^{23}$ or $\mathrm{N}_{10}$ rings in $\mathrm{BeN}_{4}{ }^{17}$ ). The most straightforward experimental way to obtain these materials could be a direct reaction between a metal, a metal nitride or an azide and nitrogen at high-pressure high-temperature (HPHT) conditions. Previous experiments with metals or metal nitrides and nitrogen in a laser-heated diamond anvil cell led to the synthesis of a variety of transition metal pernitrides $\mathrm{MN}_{2}(\mathrm{M}=\mathrm{Pt}, \mathrm{Ir}, \mathrm{Pd}$, Os, $\mathrm{Rh}, \mathrm{Ru}, \mathrm{Co}, \mathrm{Ti}$ ) with different structures: $\mathrm{Pd}$ and $\mathrm{Pt}$ pernitrides have the pyrite-type structure (cubic $\mathrm{Pa}-3)^{32,33}, \mathrm{IrN}_{2}-$ the baddelyite-type structure (monoclinic $P 2_{1} / c$ ) ${ }^{33}$, and $\mathrm{OsN}_{2}$, $\mathrm{RhN}_{2}, \mathrm{RuN}_{2}, \mathrm{CoN}_{2}$ - the marcasite-type structure (orthorhombic Pnnm $)^{34-37}$ and $\mathrm{TiN}_{2}-\mathrm{Al}_{2} \mathrm{Cu}$-type structure ${ }^{38}$. All of these pernitrides contain dinitrogen $\mathrm{N}-\mathrm{N}$ units within their structures. Due to the strong covalent $\mathrm{N}-\mathrm{N}$ bonding, many of these compounds possess exceptionally high bulk moduli suggesting potentially high hardness, which could be further enhanced, if more nitrogen would be incorporated into the structure ${ }^{31}$. For this reason many of the predicted $\mathrm{MN}_{\mathrm{x}}$ compounds are often considered not only as HEDMs, but also as possible ultra-hard low-compressible materials ${ }^{30,31}$.

In addition, extensive high-pressure investigations of alkalimetal azides $\left(\mathrm{AN}_{3}\right.$, with $\mathrm{A}=\mathrm{Li}, \mathrm{Na}, \mathrm{K}$ or $\left.\mathrm{Cs}\right)$ also aimed at nitrogen polymerization ${ }^{39-42}$. However, unambiguous structural characterization of the obtained high-pressure phases is usually hindered, as they suffer of a lack of crystallinity. Laser heating of cesium azide in a diamond anvil cell (DAC) in the of excess $\mathrm{N}_{2}$ at $60 \mathrm{GPa}$ led recently to the synthesis of a material interpreted as a cesium pentazolate salt $\mathrm{CsN}_{5}{ }^{40}$. Shortly after this discovery the first pentazolate-containing complexes were isolated at ambient pressure $^{43}$. This is a good example that information about chemistry and novel bonding of nitrogen at high-pressure may be useful for ambient-pressure synthesis.

The major challenge in the identification of products of HPHT synthesis is the absence of the information on both the chemical composition and the structure. The quality of powder X-ray diffraction data collected in DAC experiments in general is insufficient for solving the structure ab initio. Thus, interpretations of the results are often ambiguous and rely strongly on theoretical predictions.

Here, we overcame this methodological limitation mentioned above. We used laser-heated diamond anvil cells for the synthesis of ironnitrogen compounds through a direct reaction between iron and molecular nitrogen (see Methods for details). The reaction products were characterized using single-crystal X-ray diffraction, and this methodology was extended to over $130 \mathrm{GPa}$. We report three novel compounds, $\mathrm{Fe}_{3} \mathrm{~N}_{2}, \mathrm{FeN}_{2}$, and $\mathrm{FeN}_{4}$. The crystal structure of $\mathrm{FeN}_{4}$ possesses polymeric nitrogen chains that are much desired for designing potential high energy density materials. Moreover, our experimental results and theoretical analysis revealed unexpectedly complex chemical bonding in the polymeric nitrogen chains.

\section{Results and Discussion}

Synthesis and crystal structure of $\mathrm{Fe}_{3} \mathrm{~N}_{2}$. Laser heating of Fe foil in nitrogen medium at $50 \mathrm{GPa}$ and $1900(200) \mathrm{K}$ led to the formation of two nitrides $\mathrm{Fe}_{3} \mathrm{~N}_{2}$ and $\mathrm{FeN}$. Iron nitride $\mathrm{Fe}_{3} \mathrm{~N}_{2}$ is isostructural to chromium carbide $\mathrm{Cr}_{3} \mathrm{C}_{2}{ }^{44}$ (Fig. 1a). The structure is built of quadrilateral face-capped trigonal prisms $\mathrm{NFe}_{7}$, which are interconnected by sharing trigonal faces and edges. Such triangular prismatic coordination of six metal atoms about a central nonmetal atom with additional atoms situated outside the quadrilateral faces of the prism is very common for metal-rich compounds containing transition metals and elements with unfilled $p$ levels ${ }^{45,46}$. After laser-heating at pressures above $50 \mathrm{GPa}$, this phase was no longer observed.

Synthesis and crystal structure of FeN. The phase with the chemical composition $\mathrm{FeN}$ and the B8 (NiAs) structure type (Fig. 1b) was observed at each pressure-temperature point (Table 1). Very recently, FeN was reported in three independent experimental studies. Clark et al. synthesized NiAs-type FeN by heating $\mathrm{Fe}_{2} \mathrm{~N}$ in the nitrogen pressure-transmitting medium at $\sim 12 \mathrm{GPa}^{47}$, while Niwa et al. have obtained the same compound in a direct reaction between $\mathrm{Fe}$ and $\mathrm{N}_{2}$ at $\sim 35 \mathrm{GPa}^{37}$. Our experiments indicate that this compound has a wide stability range and in agreement with a recent study by Laniel et $\mathrm{al}^{48}$. On decompression, it is stable down to ambient pressure, but with time, it transforms to zincblende-structured FeN. The volume-pressure dependence for B8-FeN can be described with the third order Birch-Murnaghan equation of state with $\mathrm{V}_{0}=$ 34.03(1) $\AA^{3}, K_{0}=185(14) \mathrm{GPa}, K_{0}{ }^{\prime}=6.3$ (4) (Fig. 2a).

Synthesis and crystal structure of $\mathbf{F e N}_{2}$. The $\mathrm{FeN}_{2}$ phase was first observed after heating at $\sim 58 \mathrm{GPa}$. Iron diazenides with different crystal structures $(R \overline{3} \mathrm{~m}$ and $P n n m)$ were predicted by ab initio calculations ${ }^{49,50}$. We indeed synthesized a phase with the marcasite structure type (space group Pnnm, Fig. 1c, Table 2). The structure of $\mathrm{FeN}_{2}$ can be described as consisting of chains of edge-sharing $\mathrm{FeN}_{6}$ octahedra aligned along the $c$-axis. These chains are interconnected through common vertices. Additional linkage between $\mathrm{FeN}_{6}$ octahedra is provided via $\mathrm{N}-\mathrm{N}$ bonds (Fig. 1c). According to our structure refinement, the $\mathrm{N}-\mathrm{N}$ distance at $58.5(5) \mathrm{GPa}$ is $1.307(7) \AA$, and that is intermediate between the expected bond lengths for double and single-bonded dinitrogen units. For example, the $\mathrm{N}=\mathrm{N}$ bond in $\left[\mathrm{N}_{2}\right]^{2-}$ ions in $\mathrm{BaN}_{2}$ is of $1.23 \AA$ at ambient conditions ${ }^{51}$, whereas the calculated $\mathrm{N}-\mathrm{N}$ bond lengths in $\left[\mathrm{N}_{2}\right]^{4-}$ in $\mathrm{PtN}_{2}$ and $\mathrm{OsN}_{2}$ at ambient conditions are 1.41 and $1.43 \AA$, respectively ${ }^{52,53}$.

The compressional behavior of $\mathrm{MN}_{2}$ compounds may give an insight on the oxidation state of the metal and on the bonding between nitrogen atoms. Since, the compression of dinitrides is primarily controlled by the compression of metal-nitrogen (M-N) bonds ${ }^{34,35,37}$, the dinitrides with weaker $\mathrm{M}-\mathrm{N}$ bonds are expected to be more compressible. The strength of a M-N bond depends to a large extent on its ionicity. Therefore, the compressibility of $\mathrm{M}-\mathrm{N}$ bonds should decrease in the following sequence: $\mathrm{M}^{2+}-\mathrm{N}>$ $\mathrm{M}^{3+}-\mathrm{N}>\mathrm{M}^{4+}-\mathrm{N}$. This trend is clearly demonstrated by the experimental and theoretical studies. Metals that cannot 
a

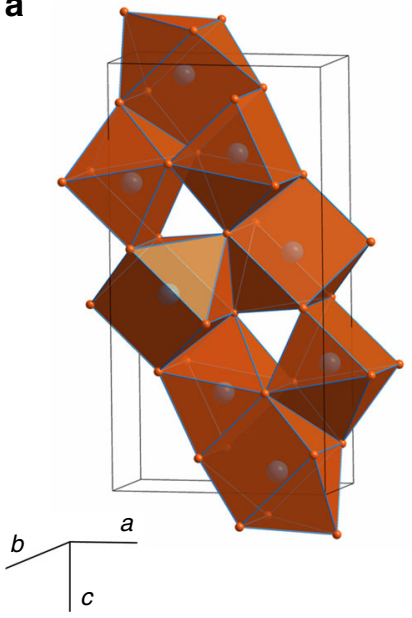

C

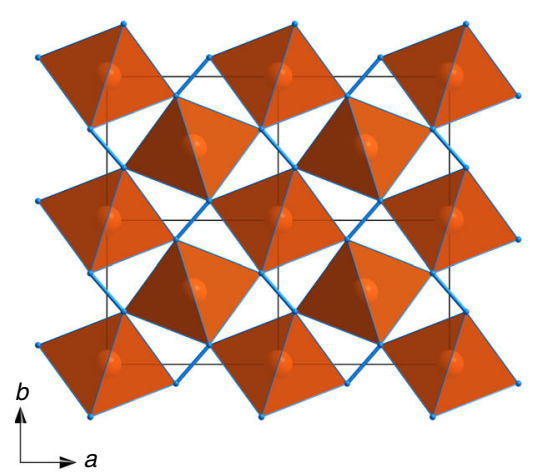

b

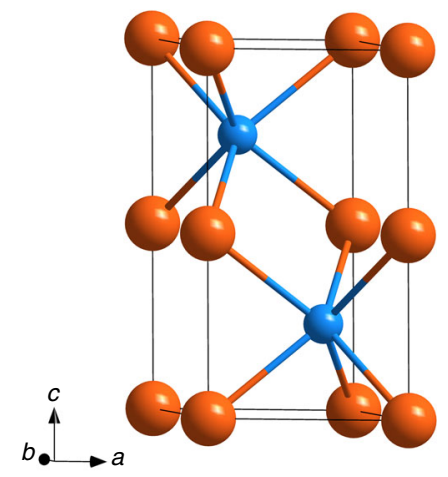

d

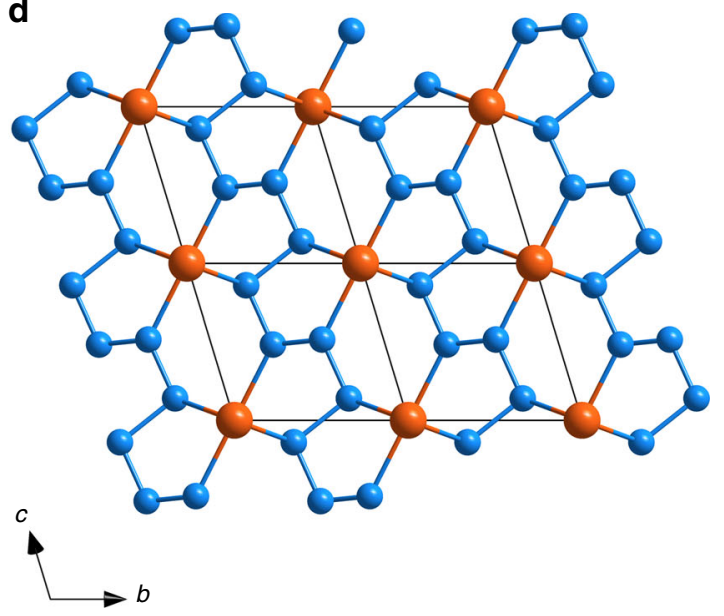

Fig. 1 Crystal structures of ironnitrogen compounds. Orange and blue balls show the positions of $\mathrm{Fe}$ and $\mathrm{N}$ atoms, respectively. a Fe $3 \mathrm{~N}_{2}$ at $50 \mathrm{GPa}$. The structure is built of quadrilateral face-capped trigonal prisms $\mathrm{NFe}_{7}$, which are interconnected by sharing trigonal faces and edges. $\mathbf{b}$ FeN at $50 \mathrm{GPa}$ with $\mathrm{NiAs}$ structure type. $\mathbf{c} \mathrm{FeN}_{2}$ at $58 \mathrm{GPa}$; Shown are the $\mathrm{FeN}_{6}$ octahedra, which are connected into infinite chains through common edges and aligned along the $c$-axis. These chains are interconnected through common vertices. Additional linkage between $\mathrm{FeN}_{6}$ octahedra is provided via $\mathrm{N}-\mathrm{N}$ bonds. $\mathbf{d}$ FeN $\mathrm{N}_{4}$ at $135 \mathrm{GPa}$. In the structure of $\mathrm{FeN}_{4}$, each Fe atom is a member of two non-planar five-member Fe[N4] metallacycles, which are almost parallel to the (1-10) lattice plane. Nitrogen atoms form infinite zigzag chains, running along the c-direction

Table 1 Summary of the experimental points at which laser-heating was performed

\begin{tabular}{|c|c|c|c|c|}
\hline Pressure before heating (GPa) & Pressure after heating & Experiment's number & Temperature (K) & Phases \\
\hline 45.2 & 49.6 & 1 & $1900 \pm 200$ & $\mathrm{Fe}_{3} \mathrm{~N}_{2}, \mathrm{FeN}$ \\
\hline 55.0 & 58.5 & 1 & $2100 \pm 200$ & $\mathrm{FeN}_{2}, \mathrm{FeN}$ \\
\hline 65.1 & 69.6 & 1 & $2200 \pm 200$ & $\mathrm{FeN}_{2}, \mathrm{FeN}$ \\
\hline $104^{a}$ & 106.0 & 2 & $>2000$ & $\mathrm{FeN}_{4}, \mathrm{FeN}$ \\
\hline $130.0^{\mathrm{a}}$ & 135.0 & 2 & $>2000$ & $\mathrm{FeN}_{4}, \mathrm{FeN}$ \\
\hline $105^{a}$ & 106.8 & 3 & $>2000$ & $\mathrm{FeN}_{4}, \mathrm{FeN}$ \\
\hline
\end{tabular}

have oxidation state larger than $+2(\mathrm{Ba}, \mathrm{Sr}, \mathrm{Ca})$, form diazenides $\mathrm{M}^{2+}[\mathrm{N}=\mathrm{N}]^{2-}$ with the $\mathrm{N}=\mathrm{N}$ distances in the range $1.2-1.24 \AA^{54-56}$ and rather low bulk moduli $\left(K_{0}\left(\mathrm{SrN}_{2}\right)=65 \mathrm{GPa}\right.$, $\left.K_{0}\left(\mathrm{BaN}_{2}\right)=46 \mathrm{GPa}\right)^{51}$. Metals that have stable oxidation states $+4(\mathrm{Os}, \mathrm{Ru}, \mathrm{Ir}, \mathrm{Ti}, \mathrm{Pt})$ form pernitrides $\mathrm{M}^{4+}[\mathrm{N}-\mathrm{N}]^{4-}$ with $\mathrm{N}-\mathrm{N}$ distances $\sim 1.4 \AA$ and are highly incompressible with very large bulk moduli $\left(K_{0}\left(\mathrm{OsN}_{2}\right)=362 \mathrm{GPa}^{52}, K_{0}\left(\mathrm{IrN}_{2}\right)=428 \mathrm{GPa}^{57}\right.$, $\left.K_{0}\left(\mathrm{TiN}_{2}\right)=385 \mathrm{GPa}^{38}, K_{0}\left(\mathrm{PtN}_{2}\right)=372 \mathrm{GPa}^{32}\right)$. Regarding the known pernitrides of those transition metals, that do not readily possess an oxidation state $+4(\mathrm{Co}, \mathrm{Rh})$, they have intermediate bulk moduli $\left(K_{0}\left(\mathrm{CoN}_{2}\right)=216 \mathrm{GPa}, K_{0}\left(\mathrm{RhN}_{2}\right)=235 \mathrm{GPa}\right)^{35,37}$, suggesting the oxidation state of $\mathrm{Co}$ and $\mathrm{Rh}$ to be +3 . According to our data, compressibility of $\mathrm{FeN}_{2}$ could be described with the $3^{\text {rd }}$ order Birch-Murnaghan equation of state with $K_{0}=250(16)$ GPa, $K_{0}{ }^{\prime}=4.0(5)$, and $V_{0}=47.42 \AA^{3}$ (Fig. 2b). Therefore, both refined $\mathrm{N}-\mathrm{N}$ distances and compressibility suggest that $\mathrm{Fe}$ in $\mathrm{FeN}_{2}$ 

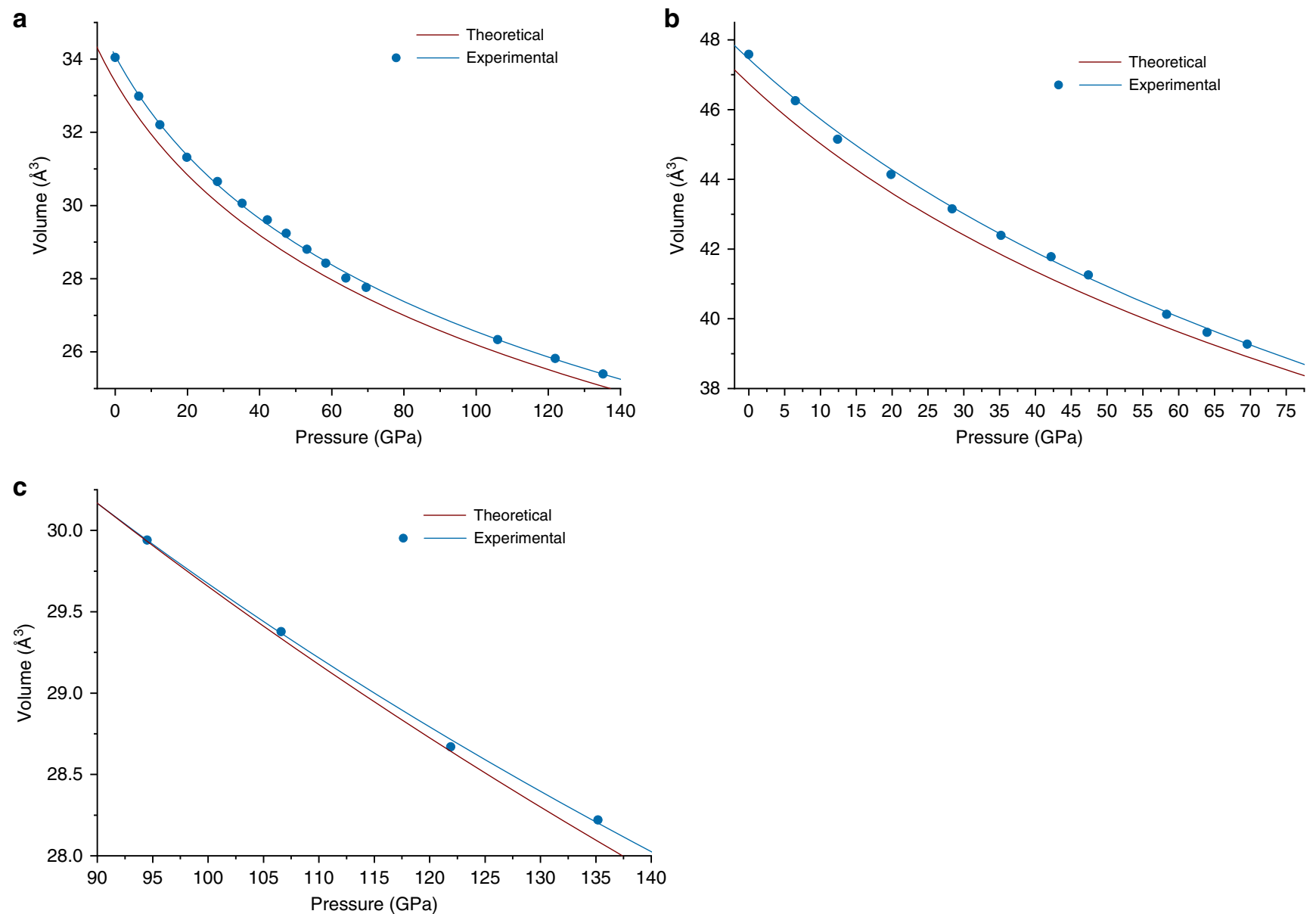

Fig. 2 Pressure dependence of the unit cell volume of ironnitrogen compounds. a FeN, $\mathbf{b}$ FeN 2 , and $\mathbf{c}$ FeN $\mathrm{N}_{4}$. Solid curves show the fit of the Birch-Murnaghan equation of state to the experimental data. $V_{0}(\mathrm{FeN})=34.03(1) \AA^{3}, K_{0}(\mathrm{FeN})=185(14) \mathrm{GPa}, K_{0}{ }^{\prime}(\mathrm{FeN})=6.3(4) ; V_{0}\left(\mathrm{FeN} \mathrm{N}_{2}\right)=47.42 \AA^{3}$, $K_{0}\left(\mathrm{FeN}_{2}\right)=250(16) \mathrm{GPa}, K_{0}{ }^{\prime}\left(\mathrm{FeN}_{2}\right)=4.0(5) ; V_{94.5}\left(\mathrm{FeN}_{4}\right)=29.94(4) \AA^{3}, K_{94.5}\left(\mathrm{FeN}_{4}\right)=603(22) \mathrm{GPa}_{1} K_{94.5}^{\prime}\left(\mathrm{FeN}_{4}\right)=4.0$ (fixed)

has an oxidation state +3 . The presence of $\left[\mathrm{N}_{2}\right]^{3 \cdot-}$ radical ions is not very likely (however, not excluded) ${ }^{56}$, and the electron count in $\mathrm{FeN}_{2}$ can be similar to that suggested for $\mathrm{LaN}_{2} \cdot{ }^{51} \mathrm{Fe}^{3+}+$ $[\mathrm{N}=\mathrm{N}]^{2-}+e^{-}$, where the electron enters the conduction band.

Synthesis and crystal structure of $\mathrm{FeN}_{4}$. The synthesis of $\mathrm{FeN}_{4}$ was first performed at $\sim 106 \mathrm{GPa}$ by laser-heating in nitrogen medium of the mixture of $\mathrm{FeN}$ and $\mathrm{FeN}_{2}$, obtained before in the reaction between $\mathrm{Fe}$ and $\mathrm{N}_{2}$ at $60 \mathrm{GPa}$ (Experiment \#2 in Table 1). The synthesis of $\mathrm{FeN}_{4}$ was reproduced in the Experiment \#3 by heating iron foil in nitrogen medium at $106 \mathrm{GPa}$. In the following discussion, we will always refer to the best-quality single-crystal XRD dataset, which was obtained at $\sim 135 \mathrm{GPa}$. The indexing of the diffraction pattern resulted in the triclinic unit cell with the parameters $a=2.5089(4), b=3.5245(13), c=3.5409(5)$ $\AA, \alpha=105.08(2), \beta=110.260(14), \gamma=92.03(2)^{\circ}$ (see Table 2, Supplementary Figs. 1-5 and Supplementary Data 4 for details). The crystal structure of the new phase was solved and refined resulting in the composition $\mathrm{FeN}_{4}$ (Figs. 1d, 3). In the structure of $\mathrm{FeN}_{4}$, six nitrogen atoms coordinate each iron atom in the following way: each $\mathrm{Fe}$ atom is a member of two non-planar fivemember $\mathrm{Fe}\left[\mathrm{N}_{4}\right]$ metallacycles, which are almost parallel to the (1-10) lattice plane (Fig. 3a, b). Two more nitrogen atoms complete the distorted octahedral coordination of Fe (Fig. 3b). The most intriguing feature of the crystal structure is displayed by nitrogen atoms forming infinite zigzag chains, running along the c-direction (Figs. 1d, 3).
The geometry of the polymeric nitrogen chains gives an insight into the electron localization within the compound. The N1 atoms have only three neighboring atoms in planar triangular geometry, whereas $\mathrm{N} 2$ atoms have tetrahedral coordination (Fig. 3c). This directly suggests the $s p^{2}$ hybridization of N1 atoms and $s p^{3}$ hybridization of $\mathrm{N} 2$ atoms. Additionally, taking in account the N1-N1, N1-N2 and N2-N2 bond distances, which at $135 \mathrm{GPa}$ equal $\sim 1.29(5), 1.30(3)$, and $1.43(4) \AA$, respectively one can classify the $\mathrm{N} 1-\mathrm{N} 1$ bonds as $\mathrm{N}=\mathrm{N}$ double bonds, and the $\mathrm{N} 1-\mathrm{N} 2$ and N2-N2 bonds as the single bonds. Moreover, theoretical analysis (see below) also shows that double N1-N1 bonds have a significantly higher electron density between atoms than single N1-N2 and N2-N2 bonds (Fig. 3d). Therefore, the nitrogen chains in $\mathrm{FeN}_{4}$ can be considered as catena-poly[tetraz1-ene-1,4-diyl] anions (Fig. 3e). The tetrazene unit $\mathrm{N}_{4}^{2-}$, thus, serves as a dianionic ligand (Fig. $3 e, f$ ), which agrees with the description of $\mathrm{Fe}$ atoms in the formal oxidation state +2 . The oxidation state +2 is also suggested by the results of Mössbauer spectroscopy (Supplementary Fig. 6). The coordination scheme of $\mathrm{Fe}$ atom in $\mathrm{FeN}_{4}$ perfectly matches the 18-electron rule (6 electrons of $\mathrm{Fe}^{2+}$ plus 12 electrons from ligands). An attempt to study $\mathrm{FeN}_{4}$ using Raman spectroscopy was not successful due to the strong fluorescence background (Supplementary Fig. 7).

To gain a deeper insight into the bonding features of $\mathrm{FeN}_{4}$, we have performed electronic structure calculations (see Methods). The Bader charge analysis, which shows the charge transfer of $0.37 e$ to $\mathrm{N} 2$ atoms and $0.25 e$ to $\mathrm{N} 1$ atoms, is in agreement with the proposed above bonding scheme. For understanding the 
Table 2 Crystallographic data for new iron Fe- $\mathbf{N}$ compounds synthesized in the present study at indicated pressures (full crystallographic information is provided in Supplementary Data 1-4)

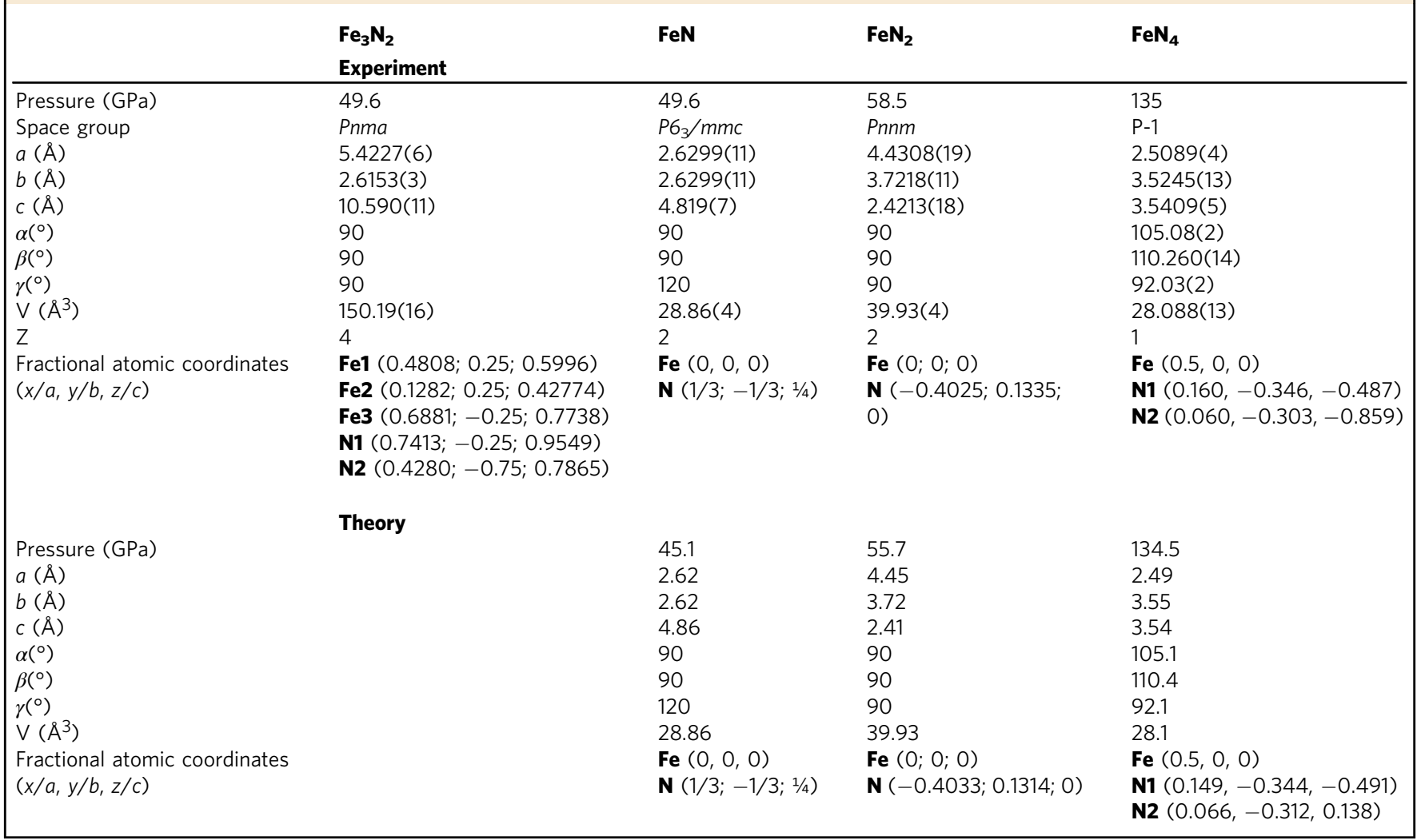

a

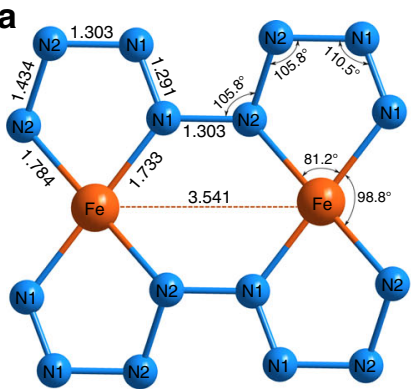

d

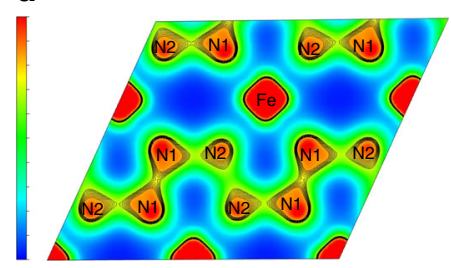

b

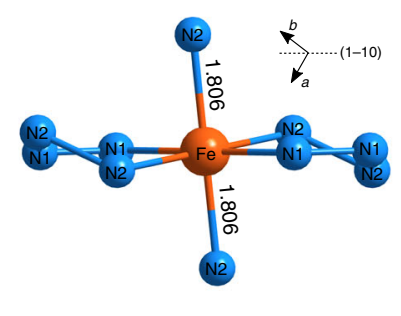

C

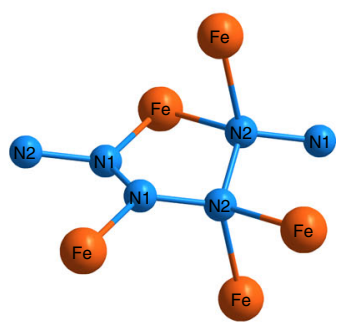

e<smiles>CC1(C)C[N+]2=[N+](C1)C(C)(C)C2</smiles>

$\mathbf{f}$

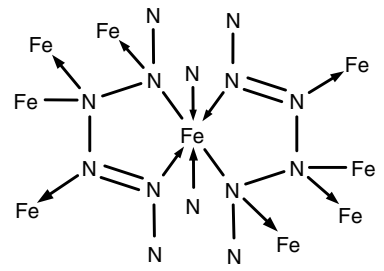

Fig. 3 Fragments of the crystal structure of $\mathrm{FeN}_{4}$ at $135 \mathrm{GPa}$. a A fragment of the crystal structure parallel to the (1-10) lattice plane featuring polymeric zigzag N-N chains. Out-off -plane atoms are not shown. $\mathbf{b}$ The same fragment shown in a different projection. $\mathbf{c} A$ fragment of the crystal structure showing the coordination geometry of the nitrogen atoms. $\mathbf{d}$ The charge density map with zig-zag $\mathrm{N}-\mathrm{N}$ chains in FeN 4 structure. e A scheme of poly[tetraz1-ene-1,4-diyl] anion. $\mathbf{f} \mathrm{A}$ scheme of coordination of iron atoms by poly[tetraz-1-ene-1,4-diyl] anions

dynamical stability of $\mathrm{FeN}_{4}$, the phonon dispersion relations were calculated at different volumes (Fig. 4). The vibrational frequencies throughout the Brillouin zone are all real, in agreement with the dynamic stability of the phase in the studied pressure range.
We were able to decompress the sample containing $\mathrm{FeN}_{4}$ obtained in the Experiment \#3 down to 22.7(2) GPa (Supplementary Fig. 5); however, the crystal quality significantly decreased and it was not possible to collect a single-crystal diffraction dataset suitable for the reliable indexing and structure 
a

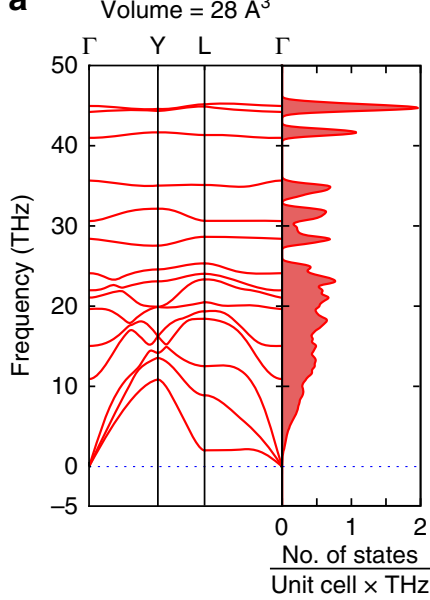

b Volume $=30 \AA^{3}$

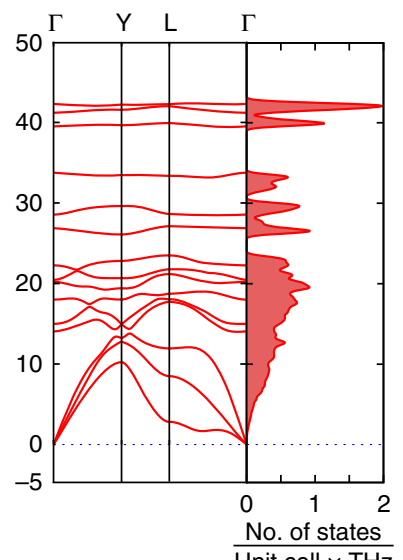

C Volume $=38 \AA^{3}$

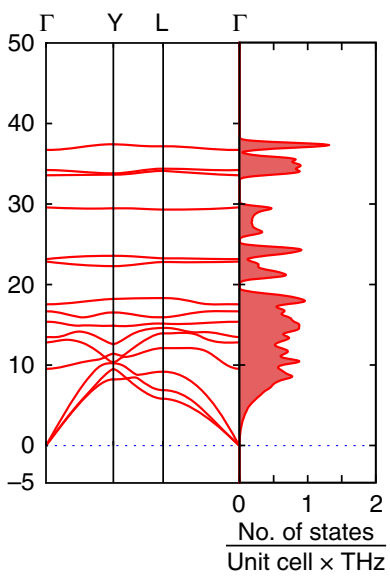

Fig. 4 Phonon dispersion curves and phonon density of states of FeN 4 . Calculations were performed with $U=4.0$ eV at different volumes, which correspond to pressures of $135 \mathrm{GPa}(\mathbf{a}), 95 \mathrm{GPa}$ (b). and $0 \mathrm{GPa}$ (c). The phonon density of states are calculated with $0.2 \mathrm{THz}$ smearing

refinement below $94.5 \mathrm{GPa}$. The pressure dependence of the unit volume data (Fig. 2c) can be described with the second-order Birch-Murnaghan equation of state with the following parameters: $K_{94.5}=603(22) \mathrm{GPa}, V_{94.5}=29.94(4) \AA^{3}$, where $K_{94.5}$ and $V_{94.5}$ are the bulk modulus and the unit cell volume at $94.5 \mathrm{GPa}$. The bulk modulus of $\mathrm{FeN}_{4}$ at $94.5 \mathrm{GPa}$ is slightly smaller than that of $\mathrm{FeN}\left(K_{94.5}(\mathrm{FeN})=679 \mathrm{GPa}\right)$. Although the attempt to recover $\mathrm{FeN}_{4}$ was unsuccessful, according to our calculations the compound should be dynamically stable even at ambient pressure (Fig. 4). Therefore, there is still a chance that $\mathrm{FeN}_{4}$ stabilization can be potentially achieved through low-temperature decompression.

Examples of transition metal complexes with tetraazadienes ${ }^{58}$, tetraaz-2-enes ${ }^{59}$ or hexazene ${ }^{60,61}$ ligands are known, and the longest cycle-containing chain has $11 \mathrm{~N}$ atoms in 1,1'-(triaz-1ene-1,3-diyl)bis(1H-tetrazol-5-amine $)^{62}$. However, we are unaware about the existence of linear nitrogen chains containing more than six nitrogen atoms, which have been reported hitherto. Interestingly, polytetrazene-type nitrogen chains were predicted to exist in the high-pressure phase with $\mathrm{N}_{2} \mathrm{H}$ stoichiometry, in $\mathrm{BeN}_{4}$ and in $\mathrm{RuN}_{4}{ }^{17,28,63}$. In these chains, the ratio of the number of single to double $\mathrm{N}-\mathrm{N}$ bonds (3:1) is the same as that we found in $\mathrm{FeN}_{4}$, however the structures are different. To the best of our knowledge, $\mathrm{FeN}_{4}$ is the first experimentally confirmed nitrogen compound with polymeric nitrogen chains.

To summarize, in the present work we synthesized a number of compounds in ironnitrogen system, solved and refined their crystal structures, and analyzed their chemical bonding. Our results contribute to both fundamental and applied science starting from fundamental understanding of nitrogen chemistry to the design of novel HEDMs. The first characterized polynitrogen compound is an important milestone for further theoretical and experimental studies. It is reasonable to suggest that compounds like $\mathrm{FeN}_{4}$ may exist in other metal-nitrogen systems and can be metastable down to ambient pressures. Such stabilizaiton can be potentially achieved through low-temperature decompression or through doping. The high synthetic pressure for $\mathrm{FeN}_{4}$ makes it hardly useful for any practical application as a HEDM at present, although its estimated volumetric energy density is $13-15.1 \mathrm{~kJ} \mathrm{~cm}^{-3}$ (see Supplementary Methods for details), which is higher than that of TNT $\left(7.2-8.0 \mathrm{~kJ} \mathrm{~cm}^{-3}\right)$, 1,3,5-trinitroperhydro-1,3,5-triazine (RDX) $\left(10.1 \mathrm{~kJ} \mathrm{~cm}^{-3}\right)$, and pentaerythritol tetranitrate $(\mathrm{PETN})\left(10.6 \mathrm{~kJ} \mathrm{~cm}^{-3}\right)^{25}$. However, the information about nitrogen bonding in this compound is important for further theoretical and experimental studies in the field.

\section{Methods}

Experiment \#1. Three pieces of iron were loaded into a BX90 diamond anvil cell equipped with $250 \mu \mathrm{m}$ Boehler-Almax diamonds. The cell was loaded with $\mathrm{N}_{2}$ as a pressure transmitting medium using the gas-loading system installed at the Bayerisches Geoinstitut. Ruby sphere was placed along with the iron pieces for pressure determination. Fe pieces were laser-heated in the DAC at 50,60, and $70 \mathrm{GPa}$ up to 1900,2100 , and $2200 \mathrm{~K}$, respectively (Table 1 ) using the portable laser-heating system at the beamline P02.2 at PETRA III ${ }^{64}$. We should note here that generally, pressure in the sample chamber increases after laser heating. Therefore, we provide pressures before and after heating in the Table 1.

Experiments \#2 and \#3. A piece of iron was placed inside a $60 \mu \mathrm{m}$ hole in a $\mathrm{Re}$ gasket, preindented to the thickness of $22 \mu \mathrm{m}$. The sample chamber was loaded with nitrogen, which served as a pressure-transmitting medium. We used BX90 diamond anvil cells equipped with Boehler-Almax type diamonds (culet diameter of $120 \mu \mathrm{m}$ ). In the Expreiment $\# 2$ the laser-heating was done at 60,106 , and $135 \mathrm{GPa}$. In the Experiment \#3, the sample was first heated at $106.8 \mathrm{GPa}$. We have used the double-sided laser-heating system of the beamline ID18 of ESRF ${ }^{64}$. In the experiment \#2, we have used ${ }^{57} \mathrm{Fe}$ as a starting material.

X-ray diffraction. The samples were studied by means of single-crystal X-ray diffraction on the synchrotron beamlines P02.2 at DESY, Hamburg, Germany $(\lambda=$ $0.2966 \AA$ A, Perkin Elmer XRD1621 flat panel detector); 13IDD at the advanced photon source (APS), Argonne, USA (MAR165 CCD detector, $\lambda=0.2952 \AA$ ) and ID27 at ESRF ( $\lambda=0.3738 \AA$, Perkin Elmer XRD1621 flat panel detector). At each pressure step, we collected the X-ray diffraction images upon continuous rotation of the cell from $-20^{\circ}$ to $+20^{\circ} \omega$. At selected pressure points, we collected the data with a narrow $0.5^{\circ}$ scanning step in the range from $-38^{\circ}$ to $+38^{\circ} \omega$.

In the experiment \# 1 we determined pressure using the fluorescence line R1 of ruby. In the experiments $\# 2$ and $\# 3$ we determined pressures using the equations of state of $h c p-\mathrm{Fe}$ and/or Re.

Whereas the starting material, a polycrystalline iron foil, gives characteristic Debye-Scherer rings in the diffraction pattern, after the laser-heating in solidified nitrogen, we clearly observed well defined, sharp diffraction spots from multiple grains of new high-pressure phases. Using the Ewald ${ }^{\text {Pro }}$ reciprocal space viewing tool for the CrysAlis ${ }^{\text {Pro }}$ program ${ }^{65}$, we were able to identify the diffraction spots belonging to certain domains, find their orientation matrices and refine the unit cell parameters. The structures of the new phases were solved against single-crystal diffraction data. The general procedure of the analysis of a multigrain diffraction dataset is described in ref. ${ }^{66}$. We provide several raw diffraction images with grain indexing examples in the Supplementary Figs. 1-4 and Supplementary Note 1. Further discussion regarding indexing solutions is given in the Peer Review file.

Diffraction data analysis (peak search, unit cell finding, data integration, frame scaling etc.) was performed with CrysAlis ${ }^{\text {Pro }}$ software. The crystal structures were solved using the computer program SHELXT that employs a dual-space algorithm for the solution of a phase problem ${ }^{67}$. General output of the structure solution program was a position of heavy iron atom, while nitrogen atoms were located based on the analysis of residual electron density maps. Crystal structures were refined against single-crystal diffraction data using the computer program JANA2006 $6^{68}$ (see Supplementary Data 1-9 for structural details). The obtained 
models represent the authors' optimum refinement of the available X-ray data. The full diffraction data are made available online (see Data availability section). Their complexity for processing is obvious due to the reduced data to parameter ratio, which is characteristic for all single-crystal diffraction data sets obtained in a DAC, and due to the presence of diffraction from numerous domains. If improvements in data processing become available, one can use the present data for reevaluation.

Calculations. The ab-initio calculations were performed using the supercell technique and all electron projector-augmented-wave (PAW) method ${ }^{69}$ as implemented in the VASP code $^{70-72}$. The simulations were carried out using 4-atoms (B8-FeN), 5-atoms $\left(\mathrm{FeN}_{4}\right)$ and 6-atoms periodic $\left(\mathrm{FeN}_{2}\right)$ cells. The integration over the Brillouin zone is performed using the Gamma scheme with $29 \times 29 \times 29 \mathrm{k}$ point grids for B8-FeN and $18 \times 18 \times 18$ k-point grids for $\mathrm{FeN}_{2}$ and $\mathrm{FeN}_{4}$ structures. Gaussian smearing method was chosen with a smearing width of $0.05 \mathrm{eV}$. The energy cutoff for the plane waves included in the expansion of wave functions was set to $500 \mathrm{eV}$. The convergence criterion for the electronic subsystem was chosen to be equal to $10^{-4} \mathrm{eV}$ for two subsequent iterations, and the ionic relaxation loop within the conjugated gradient method was stopped when forces became of the order of $10^{-3} \mathrm{eV} / \AA$.

The exchange-correlation energy was described using the Perdew-Wang-91 GGA functional ${ }^{73}$ augmented by including Hubbard-U corrections within the DFT $+U$ method following the Dudarev's approach ${ }^{74}$. The chosen parameters $\mathrm{U}=4.0 \mathrm{eV}$ and $\mathrm{J}=1 \mathrm{eV}$ for the $\mathrm{Fe} d$ states provide good agreement with the experimental structural characteristics for all simulated systems $\mathrm{FeN}, \mathrm{FeN}_{2}$, and $\mathrm{FeN}_{4}$ structures (Fig. 2, Table 2). We found that $\mathrm{FeN}$ is magnetic and used ferromagnetic configuration in our simulations. $\mathrm{FeN}_{2}$, and $\mathrm{FeN}_{4}$ were found to be non-magnetic.

Bader charge analysis ${ }^{75}$ derived from topological consideration on the charge distribution was performed using the code developed by Henkelman and colleagues $^{76}$ for $400 \times 400 \times 400 \mathrm{NG}(\mathrm{X}, \mathrm{Y}, \mathrm{Z}) \mathrm{F}$ mesh. The phonon calculations were carried out at $\mathrm{T}=0 \mathrm{~K}$ within quasi-harmonic approximation. We used a finite distortions approach implemented into the PHONOPY program 77 combined with Quantum Espresso $(\mathrm{QE})^{78}$ simulations. In the $\mathrm{QE}$ calculations, we used plane waves with kinetic energy up to $50 \mathrm{Ry}$ for the electron wave functions while the augmented charges were described using 500 Ry energy cut-off. With these optimized parameters, we reproduced the results of static calculations obtained by VASP. Converged phonon dispersions were achieved using a $(4 \times 4 \times 4)$ supercell with 320 atoms and $(4 \times 4 \times 4)$ Monkhorst-Pack ${ }^{79}$ sampling of the Brillouin zone.

Data availability. The details of the crystal structure investigations may be obtained from FIZ Karlsruhe, 76344 Eggenstein-Leopoldshafen, Germany (fax: +49-7247-808-666; e-mail: crysdata@fiz-karlsruhe.de) on quoting the deposition numbers CSD-434274-434277. Single-crystal X-ray diffraction dataset for $\mathrm{FeN}_{4}$ at $135 \mathrm{GPa}$ has been deposited to Figshare (https://figshare.com/) with the accession link https://doi.org/10.6084/m9.figshare.6471092.vl. The data that support the findings of this study are available from the corresponding author upon reasonable request.

Received: 8 December 2017 Accepted: 19 June 2018

Published online: 16 July 2018

\section{References}

1. Eremets, M. I., Gavriliuk, A. G., Trojan, I. A., Dzivenko, D. A. \& Boehler, R. Single-bonded cubic form of nitrogen. Nat. Mater. 3, 558-563 (2004).

2. Sun, W. et al. Thermodynamic routes to novel metastable nitrogen-rich nitrides. Chem. Mater. 29, 6936-6946 (2017).

3. Zarko, V. E. Searching for ways to create energetic materials based on polynitrogen compounds (review). Combust. Explos. Shock Waves 46, 121-131 (2010).

4. Zhang, L., Wang, Y., Lv, J. \& Ma, Y. Materials discovery at high pressures. Nat. Rev. Mater. 2, 17005 (2017).

5. Li, Y.-C. et al. 1,1'-Azobis-1,2,3-triazole: a high-nitrogen compound with stable $\mathrm{N}_{8}$ structure and photochromism. J. Am. Chem. Soc. 132, 12172-12173 (2010).

6. Qi, C. et al. A novel stable high-nitrogen energetic material: 4,4'-azobis(1,2,4triazole). J. Mater. Chem. 21, 3221 (2011).

7. Klapötke, T. M. \& Piercey, D. G. 1,1'-Azobis(tetrazole): a highly energetic nitrogen-rich compound with a $\mathrm{N}_{10}$ chain. Inorg. Chem. 50, 2732-2734 (2011).

8. Luo, Y.-R. Comprehensive Handbook of Chemical Bond Energies. (CRC Press, Boca Raton 2007).

9. Pickard, C. J. \& Needs, R. J. High-pressure phases of nitrogen. Phys. Rev. Lett. 102, 1-4 (2009).

10. Wang, X. et al. Cagelike diamondoid nitrogen at high pressures. Phys. Rev. Lett. 109, 1-5 (2012).
11. Ma, Y., Oganov, A. R., Li, Z., Xie, Y. \& Kotakoski, J. Novel high pressure structures of polymeric nitrogen. Phys. Rev. Lett. 102, 100-103 (2009).

12. Wang, H. et al. Nitrogen backbone oligomers. Sci. Rep. 5, 13239 (2015).

13. Goncharov, A. F. et al. Backbone $\mathrm{NxH}$ compounds at high pressures. J. Chem. Phys. 142, 214308 (2015)

14. Prasad, D. L. V. K., Ashcroft, N. W. \& Hoffmann, R. Evolving structural diversity and metallicity in compressed lithium azide. J. Phys. Chem. C 117, 20838-20846 (2013).

15. Zhang, M., Yan, H., Wei, Q., Wang, H. \& Wu, Z. Novel high-pressure phase with pseudo-benzene "N 6" molecule of LiN 3. Europhys. Lett. 101, 26004 (2013).

16. Shen, Y. et al. Novel lithium-nitrogen compounds at ambient and high pressures. Sci. Rep. 5, 14204 (2015).

17. Zhang, S., Zhao, Z., Liu, L. \& Yang, G. Pressure-induced stable BeN4 as a high-energy density material. J. Power Sources 365, 155-161 (2017).

18. Wei, S. et al. A novel polymerization of nitrogen in beryllium tetranitride at high pressure. J. Phys. Chem. C. 121, 9766-9772 (2017).

19. Steele, B. A. \& Oleynik, I. I. Sodium pentazolate: a nitrogen rich high energy density material. Chem. Phys. Lett. 643, 21-26 (2016).

20. Yu, S. et al. Emergence of novel polynitrogen molecule-like species, covalent chains and layers in magnesium-nitrogen $\mathrm{Mg}_{\mathrm{x}} \mathrm{N}_{\mathrm{y}}$ phases under high pressure. J. Phys. Chem. C. 121, 11037-11046 (2017).

21. Wei, S. et al. Alkaline-earth metal $(\mathrm{Mg})$ polynitrides at high pressure as possible high-energy materials. J. Phys. Chem. Chem. Phys. 19, 9246-9252 (2017).

22. Liu, Z. et al. Bonding properties of aluminum nitride at high pressure. Inorg. Chem. 56, 7494-7500 (2017).

23. Steele, B. A. \& Oleynik, I. I. Novel potassium polynitrides at high pressures. J. Phys. Chem. A 121, 8955-8961 (2017).

24. Zhu, S. et al. Stable calcium nitrides at ambient and high pressures. Inorg. Chem. 55, 7550-7555 (2016).

25. Zhang, J., Oganov, A. R., Li, X. \& Niu, H. Pressure-stabilized hafnium nitrides and their properties. Phys. Rev. B 95, 020103 (2017).

26. Kvashnin, A. G., Oganov, A. R., Samtsevich, A. I. \& Allahyari, Z. Computational search for novel hard chromium-based materials. J. Phys. Chem. Lett. 8, 755-764 (2017)

27. Wang, X. et al. Layered polymeric nitrogen in $\mathrm{RbN} 3$ at high pressures. Sci. Rep. 5, 16677 (2015).

28. Zhang, Y. et al. Diverse ruthenium nitrides stabilized under pressure: a theoretical prediction. Sci. Rep. 6, 33506 (2016).

29. Peng, F., Han, Y., Liu, H. \& Yao, Y. Exotic stable cesium polynitrides at high pressure. Sci. Rep. 5, 16902 (2015).

30. Aydin, S., Ciftci, Y. O. \& Tatar, A. Superhard transition metal tetranitrides: XN4 (X=Re, Os, W). J. Mater. Res. 27, 1705-1715 (2012).

31. Zhao, Z. et al. Nitrogen concentration driving the hardness of rhenium nitrides. Sci. Rep. 4, 4797 (2014).

32. Crowhurst, J. C. et al. Synthesis and characterization of the nitrides of platinum and iridium. Science 311, 1275-1278 (2006).

33. Crowhurst, J. C. et al. Synthesis and characterization of nitrides of iridium and palladium. J. Mater. Res. 23, 1-5 (2008).

34. Niwa, K. et al. Discovery of the last remaining binary platinum-group pernitride RuN2. Chemistry 20, 13885-13888 (2014).

35. Niwa, K. et al. High pressure synthesis of marcasite-type rhodium pernitride. Inorg. Chem. 53, 697-699 (2014).

36. Tse, J. S. et al. Structural phase transition in $\mathrm{Ca} \mathrm{H} 2$ at high pressures. Phys. Rev. B 75, 134108 (2007).

37. Niwa, K. et al. Highly coordinated iron and cobalt nitrides synthesized at high pressures and high temperatures. Inorg. Chem. 56, 6410-6418 (2017).

38. Bhadram, V. S., Kim, D. Y. \& Strobel, T. A. High-pressure synthesis and characterization of incompressible titanium pernitride. Chem. Mater. 28, 1616-1620 (2016).

39. Eremets, M. I. et al. Polymerization of nitrogen in sodium azide. J. Chem. Phys. 120, 10618-10623 (2004).

40. Steele, B. A. et al. High-pressure synthesis of a pentazolate salt. Chem. Mater. 29, 735-741 (2017).

41. Medvedev, S. A. et al. Phase stability of lithium azide at pressures up to 60 GPa. J. Phys. Condens. Matter 21, 195404-195405 (2009).

42. Hou, D. et al. Series of phase transitions in cesium azide under high pressure studied by in situ x-ray diffraction. Phys. Rev. B - Condens. Matter Mater. Phys. 84, 1-7 (2011).

43. Zhang, C., Sun, C., Hu, B., Yu, C. \& Lu, M. Synthesis and characterization of the pentazolate anion cyclo $-\mathrm{N}_{5}{ }^{-}$in $(\mathrm{N} 5) 6\left(\mathrm{H}_{3} \mathrm{O}\right) 3\left(\mathrm{NH}_{4}\right) 4 \mathrm{Cl}$. Science 355, 374-376 (2017).

44. Glaser, J., Schmitt, R. \& Meyer, H. J. Neue Strukturverfeinerung und Eigenschaften von $\mathrm{Cr}_{3} \mathrm{C}_{2}$. Z. Naturforsch. 58, 929-933 (2003).

45. Rundqvist, S. \& Runnsjö, G. Crystal structure refinement of $\mathrm{Cr}_{3} \mathrm{C}_{2}$. Acta Chem. Scand. 23, 1191-1199 (1969).

46. Lundström, T., Folkmarson, L., Olofsson, B. \& Nielsen, P. H. The crystal structure of $\mathrm{Hf}_{3} \mathrm{P}_{2}$. Acta Chem. Scand. 22, 2191-2199 (1968). 
47. Clark, W. P. et al. High-pressure NiAs-type modification of FeN. Angew. Chem. Int. Ed. 56, 7302-7306 (2017).

48. Laniel, D., Dewaele, A., Anzellini, S. \& Guignot, N. Study of the iron nitride FeN into the megabar regime. J. Alloy. Compd. 733, 53-58 (2018).

49. Wessel, M. \& Dronskowski, R. A new phase in the binary iron nitrogen system? The prediction of iron pernitride, $\mathrm{FeN}_{2}$. Chemistry 17, 2598-2603 (2011).

50. Wang, Z. et al. Prediction and characterization of the marcasite phase of iron pernitride under high pressure. J. Alloy. Compd. 702, 132-137 (2017).

51. Wessel, M. \& Dronskowski, R. Nature of N-N bonding within high-pressure noble-metal pernitrides and the prediction of lanthanum pernitride. J. Am. Chem. Soc. 132, 2421-2429 (2010).

52. Chen, Z. W. et al. Crystal structure and physical properties of $\mathrm{OsN}_{2}$ and $\mathrm{PtN}_{2}$ in the marcasite phase. Phys. Rev. B - Condens. Matter Mater. Phys. 75, 1-4 (2007).

53. Montoya, J. A., Hernandez, A. D., Sanloup, C., Gregoryanz, E. \& Scandolo, S. $\mathrm{OsN}_{2}$ : crystal structure and electronic properties. Appl. Phys. Lett. 90, 2005-2008 (2007).

54. Vajenine, G. V. et al. Preparation, crystal structure, and properties of barium pernitride, $\mathrm{BaN}_{2}$. Inorg. Chem. 40, 4866-4870 (2001).

55. Auffermann, G., Prots, Y. \& Kniep, R. $\mathrm{SrN}$ and $\mathrm{SrN}_{2}$ : diazenides by synthesis under High $\mathrm{N}_{2}$-pressure. Angew. Chem. Int. Ed. 40, 547-549 (2001).

56. Schneider, S. B. et al. High-pressure synthesis and characterization of $\mathrm{Li}_{2} \mathrm{Ca}_{3}\left[\mathrm{~N}_{2}\right]_{3}-$ an uncommon metallic diazenide with $\left[\mathrm{N}_{2}\right]^{2-}$ ions. J. Am. Chem. Soc. 135, 16668-16679 (2013).

57. Young, A. F. et al. Synthesis of novel transition metal nitrides $\mathrm{IrN}_{2}$ and $\mathrm{OsN}_{2}$. Phys. Rev. Lett. 96, 1-4 (2006).

58. Doedens, R. J. Molecular configuration of $(\mathrm{Me})_{2} \mathrm{~N}_{4} \mathrm{Fe}(\mathrm{CO})_{3}$, a tetrazadiene-tricarbonyliron complex. Chem. Commun. 1950, 1271-1272 (1968).

59. Vaddypally, S., McKendry, I. G., Tomlinson, W., Hooper, J. P. \& Zdilla, M. J. Electronic structure of manganese complexes of the redox-non-innocent tetrazene ligand and evidence for the metal-azide/imido cycloaddition intermediate. Chemistry 22, 10548-10557 (2016).

60. Gondzik, S. et al. Reactions of a $\mathrm{Zn}(\mathrm{I})$ complex with group 14 azidesformation of zinc azide and zinc hexazene complexes. Chem. Commun. 50, 927-929 (2014).

61. Bellow, J. A., Martin, P. D., Lord, R. L. \& Groysman, S. Reductive coupling of azides mediated by an iron(II) bis(alkoxide) complex. Inorg. Chem. 52, 12335-12337 (2013)

62. Tang, Y. et al. Synthesis and characterization of a stable, catenated $\mathrm{N}_{11}$ energetic salt. Angew. Chem. Int. Ed. 52, 4875-4877 (2013).

63. Yin, K., Wang, Y., Liu, H., Peng, F. \& Zhang, L. N. 2H: a novel polymeric hydronitrogen as a high energy density material. J. Mater. Chem. A 3, 4188-4194 (2015).

64. Kupenko, I. et al. Portable double-sided laser-heating system for Mössbauer spectroscopy and X-ray diffraction experiments at synchrotron facilities with diamond anvil cells. Rev. Sci. Instrum. 83, 124501 (2012).

65. CrysAlisPro Software system, version 1.171.37.35. Rigaku Corporation, Oxford, UK (2015).

66. Bykova, E. Single-crystal X-ray Diffraction at Extreme Conditions in Mineral Physics and Material Sciences. Doctoral thesis, University of Bayreuth (2015).

67. Sheldrick, G. M. SHELXT-Integrated space-group and crystal-structure determination. Acta Crystallogr. Sect. A Found. Adv. 71, 3-8 (2015).

68. Petricek, V., Dusek, M. \& Palatinus, L. Crystallographic computing system JANA2006: general features. Z. für Krist. 229, 345-352 (2014).

69. Blöchl, P. E. Projector augmented-wave method. Phys. Rev. B 50, 17953-17979 (1994).

70. Kresse, G. \& Furthmüller, J. Efficiency of ab-initio total energy calculations for metals and semiconductors using a plane-wave basis set. Comput. Mater. Sci. 6, 15-50 (1996).

71. Kresse, G. \& Furthmüller, J. Efficient iterative schemes for ab initio totalenergy calculations using a plane-wave basis set. Phys. Rev. B 54, 11169-11186 (1996).

72. Kresse, G. \& Joubert, D. From ultrasoft pseudopotentials to the projector augmented-wave method. Phys. Rev. B 59, 1758-1775 (1999).

73. Perdew, J. P. \& Wang, Y. Accurate and simple analytic representation of the electron-gas correlation energy. Phys. Rev. B 45, 13244-13249 (1992).

74. Dudarev, S. L., Botton, G. A., Savrasov, S. Y., Humphreys, C. J. \& Sutton, A. P. Electron-energy-loss spectra and the structural stability of nickel oxide: an LSDA+U study. Phys. Rev. B 57, 1505-1509 (1998).

75. Bader, R. F. W. Atoms in Molecules: A Quantum Theory. (Clarendon Press, Oxford 1994).

76. Sanville, E., Kenny, S. D., Smith, R. \& Henkelman, G. Improved grid-based algorithm for Bader charge allocation. J. Comput. Chem. 28, 899-908 (2007).
77. Togo, A. \& Tanaka, I. First principles phonon calculations in materials science. Scr. Mater. 108, 1-5 (2015).

78. Giannozzi, P. et al. QUANTUM ESPRESSO: a modular and open-source software project for quantum simulations of materials. J. Phys. Condens. Matter 21, 395502 (2009).

79. Monkhorst, H. J. \& Pack, J. D. Special points for Brillouin-zone integrations. Phys. Rev. B 13, 5188-5192 (1976).

80. Akahama, Y. \& Kawamura, H. Pressure calibration of diamond anvil Raman gauge to $310 \mathrm{GPa}$. J. Appl. Phys. 100, 043516 (2006).

\section{Acknowledgements}

Parts of this research were carried out at the beamline P02.2 at DESY, a member of the Helmholtz Association (HGF). Portions of this work were performed at GeoSoilEnviroCARS (The University of Chicago, Sector 13), Advanced Photon Source (APS), Argonne National Laboratory. GeoSoilEnviroCARS is supported by the National Science Foundation-Earth Sciences (EAR-1634415) and Department of Energy- GeoSciences (DE-FG02-94ER14466). This research used resources of the Advanced Photon Source, a U.S. Department of Energy (DOE) Office of Science User Facility operated for the DOE Office of Science by Argonne National Laboratory under Contract No. DE-AC0206CH11357. We acknowledge the European Synchrotron Radiation Facility for provision of synchrotron radiation facilities (beamlines ID18 and ID27). N.D. and L.D. thank the Deutsche Forschungsgemeinschaft (DFG projects DU 954-11/1 and DU 393-10/1) and the Federal Ministry of Education and Research, Germany (BMBF, Grant No. $5 \mathrm{~K} 16 \mathrm{WC1}$ ) for financial support. F.T. and I.A.A are grateful to the support provided by the Swedish Research Council project No 2015-04391 and the VINN Excellence Center Functional Nanoscale Materials (FunMat-2) Grant 2016-05156. Support from the Swedish Government Strategic Research Areas in Materials Science on Functional Materials at Linköping University (Faculty Grant SFO-Mat-LiU No 2009-00971) and the Swedish e-Science Research Centre (SeRC) is gratefully acknowledged. Theoretical analysis of structural properties was supported by the Ministry of Education and Science of the Russian Federation (Grant No. 14.Y26.31.0005). Simulations of the electronic structure were supported by the Ministry of Education and Science of the Russian Federation in the framework of Increase Competitiveness Program of NUST "MISIS" (No. K2-2017-080) implemented by a governmental decree dated 16 March 2013, No. 211. Calculations have been carried out at the Swedish National Infrastructure for Computing (SNIC) and at computer cluster at NUST "MISIS". Analysis of the charge density distribution was supported by the RFBR research project No 16-02-00797

\section{Author contributions}

M.B, L.D, N.D., and I.A. wrote the manuscript, M.B., E.B., G.A., E.K., K.G., I.C., M.M., V. P. and H.P.L. performed the experiments, M.B. analyzed and interpreted the diffraction data, I.K. and C. McC. performed and interpreted Mössbauer experiments, F.T., A.P. and I.A. performed the calculations.

\section{Additional information}

Supplementary Information accompanies this paper at https://doi.org/10.1038/s41467 018-05143-2.

Competing interests: The authors declare no competing interests.

Reprints and permission information is available online at http://npg.nature.com/ reprintsandpermissions/

Publisher's note: Springer Nature remains neutral with regard to jurisdictional claims in published maps and institutional affiliations.

Open Access This article is licensed under a Creative Commons Attribution 4.0 International License, which permits use, sharing, adaptation, distribution and reproduction in any medium or format, as long as you give appropriate credit to the original author(s) and the source, provide a link to the Creative Commons license, and indicate if changes were made. The images or other third party material in this article are included in the article's Creative Commons license, unless indicated otherwise in a credit line to the material. If material is not included in the article's Creative Commons license and your intended use is not permitted by statutory regulation or exceeds the permitted use, you will need to obtain permission directly from the copyright holder. To view a copy of this license, visit http://creativecommons.org/ licenses/by/4.0/.

(c) The Author(s) 2018 